adult erythroid cells. Transl Res. 2015;165(1):115-125

4. Suzuki M, Yamamoto M, Engel JD. Fetal globin gene repressors as drug targets for molecular therapies to treat the $\beta$-globinopathies. Mol Cell Biol. 2014;34(19):3560-3569.

5. Lavelle D, Engel JD, Saunthararajah Y. Fetal hemoglobin induction by epigenetic drugs. Semin Hematol. 2018;55(2):60-67.

6. Yu X, Azzo A, Bilinovich SM, et al. Disruption of the MBD2-NuRD complex but not MBD3-NuRD induces high level HbF expression in human erythroid cells. Haematologica 2019;104(12):2361-2371.

7. Le Guezennec X, Vermeulin M, Brinkman AB, et al. MBD2/NURD and MBD3/NURD, two distinct complexes with different biochemical and functional properties Mol Cell Biol. 2006;26(3):843-851.

8. Van der Ploeg LH, Flavell RA. DNA methylation in the human gamma delta beta-globin locus in erythroid and nonerythroid tissues. Cell. 1980;19(4):947-958.

9. DeSimone J, Heller P, Hall L, Zwiers D. 5-azacytidine stimulates fetal hemoglobin synthesis in anemic baboons. Proc Natl Acad Sci U S A. 1982;79(14):4428-4431.

10. Ginder GD, Whitters MJ, Pohlman JK. Activation of a chicken embryonic globin gene in adult erythroid cells by 5 -azacytidine and sodium butyrate. Proc Natl Acad Sci USA. 1984;81(13):3954-3958.

11. Vinjamur DS, Bauer DE, Orkin SH. Recent progress in understanding and manipulating haemoglobin witching for the haemoglobinopathies. Br J Haematol. 2018;180(5):630-643.

12. Wienert B, Martyn GE, Funnell APW, Quinlan KGR, Crossley M. Wake-up sleepy gene: Reactivating fetal globin for $\beta$-hemoglobinopathies. Trends Genet. 2018;34(12):927-940.
13. Cui S, Kolodziej KE, Obara N, et al. Nuclear receptors TR2 and TR4rcruit multiple epigenetic transcriptional corepressors that associate specifically with the embryonic $\beta$-type globin promoters in differentiated adult erythroid cells. Mol Cell Biol. 2011;31(16):32983311

14. Xu J, Bauer DE, Kerenyi MA, et al. Corepressor-dependent silencing of fetal hemoglobin expression by BCL11A. Proc Natl Acad Sci U S A. 2013;110(16):6518-6523.

15. Du Q, Luu P-L, Stirzaker C, Clark SJ. Methyl-CpG-binding domain proteins:readers of the epigenome. Epigenomics. 2015;7(6):10511073

16. Menafra R, Stunnenberg HG. MBD2 and MBD3: elusive functions and mechanisms. Front Genet. 2014;5:428.

17. Rupon JW, Wang SZ, Gaensler K, Lloyd J, Ginder GD. Methyl binding domain protein 2 mediates gamma-globin gene silencing in adult human betaYAC transgenic mice. Proc Natl Acad Sci U S A. 2006;103(17):6617-6622

18. Kransdorf EP, Wang SZ, Zhu SZ, Langston TB, Rupon JW, Ginder GD. MBD2 is a critical component of a methyl cytosine-binding protein complex isolated from primary erythroid cells. Blood. 2006;108(8):2836-2845.

19. Hendrich B, Guy J, Ramsahoye B, Wilson VA, Bird A. Closely related proteins MBD2 and MBD3 play distinctive but interacting roles in mouse development. Genes Dev. 2001;15(6):710-723.

20. Sher F, Hossain M, Seruggia D, et al. Rational targeting of a NuRD subcomplex guided by comprehensive in situ mutagenesis. Nat Genet. 2019;51(7):1149-1159.

\title{
Are we ready to use precision medicine in chronic myeloid leukemia practice?
}

\author{
Dennis Dong Hwan Kim
}

Leukemia Program, Department of Medical Oncology and Hematology, Princess Margraret Cancer Centre, University Health Network, University of Toronto, Toronto, ON, Canada

E-mail: DENNIS DONG HWAN KIM - dr.dennis.kim@uhn.ca

doi:10.3324/haematol.2019.231753

○ ver the last two decades, the introduction of tyrosine kinase inhibitors (TKI) and advances in $B C R$ $A B L 1$ monitoring using quantitative polymerase chain reaction (qPCR) have significantly improved treatment outcomes in chronic myeloid leukemia (CML) patients. ${ }^{1}$ Not only the introduction of TKI increased the life expectancy of CML patients $(98 \%$ of age-matched healthy control), but also the incorporation of $B C R-A B L 1$ monitoring using qPCR significantly improved outcomes of CML patients by identifying those cases developing TKI failure and progressing to the advanced phase ${ }^{2,3}$ However, it is still challenging to predict patients at high risk for TKI failure at initial diagnosis of CML before commencing TKI therapy. Thus, major challenges still remain, including lack of accurate risk stratification at initial diagnosis.

The current algorithm for CML management is mainly based on monitoring $B C R-A B L 1$ using $\mathrm{qPCR} .{ }^{3}$ Despite its good performance, there are still remaining issues some of which include: i) how to select upfront TKI drug in a newly diagnosed CML patient (imatinib vs. newer generation TKI); ii) how to switch TKI therapy in a patient who developed TKI resistance, but without ABL1 kinase domain mutations; and iii) how to predict which patients are at high risk of progression to blastic crisis. Thus, there is an urgent demand for novel biomarkers in managing CML beyond monitoring $B C R-A B L 1$ fusion transcripts. Given this, how can we go forward from here?
Let us look back at routine CML practice 20 years ago when TKI therapy and qPCR-based $B C R$ - $A B L 1$ monitoring were not available. ${ }^{4}$ When a patient was newly diagnosed with chronic phase CML, the first step would be the identification of an HLA-matched donor for allogeneic hematopoietic cell transplantation (HCT) and co-ordination of allogeneic HCT within two years from initial diagnosis before the patient progressed to advanced phase. If an appropriate donor was not available, interferon therapy was a treatment of choice. Disease monitoring was mainly based on the metaphase cytogenetic test for which bone marrow aspiration should be performed every 6 months to assess cytogenetic response. Let us compare it with current CML practice, which has changed significantly over the last two decades. First, we no longer initiate a search for an HLA-matched donor search until TKI failure or intolerance to more than two TKI is suspected. ${ }^{3}$ Bone marrow examination does not need to be repeated as frequent as $B C R$ $A B L 1$ qPCR on peripheral blood which is the mainstay of disease monitoring. So, what will happen in the future? CML practice will evolve and will be transformed again from the current routine practice. However, what we do not know yet is how this will be achieved and what changes will be applied.

Precision medicine is becoming the mainstream of future medicine. It has been implemented in the clinical practice in acute myeloid leukemia (AML), ${ }^{5}$ and myeloproliferative 
A

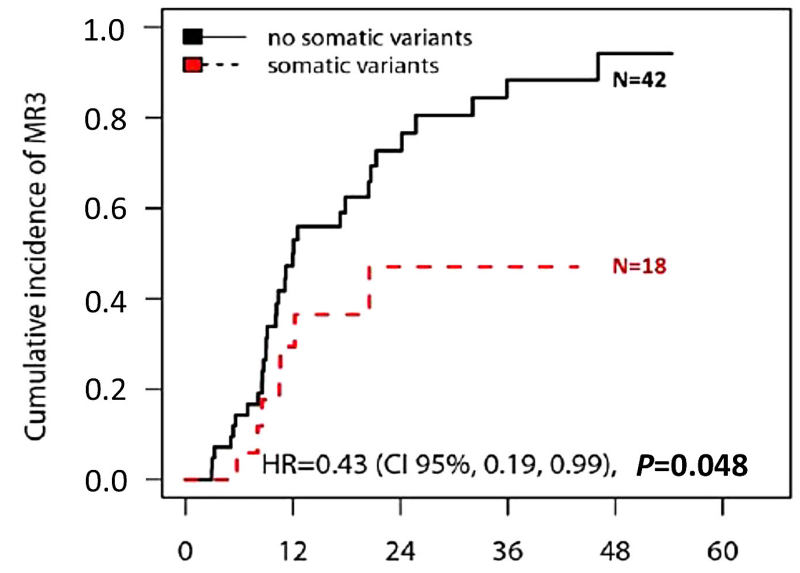

B

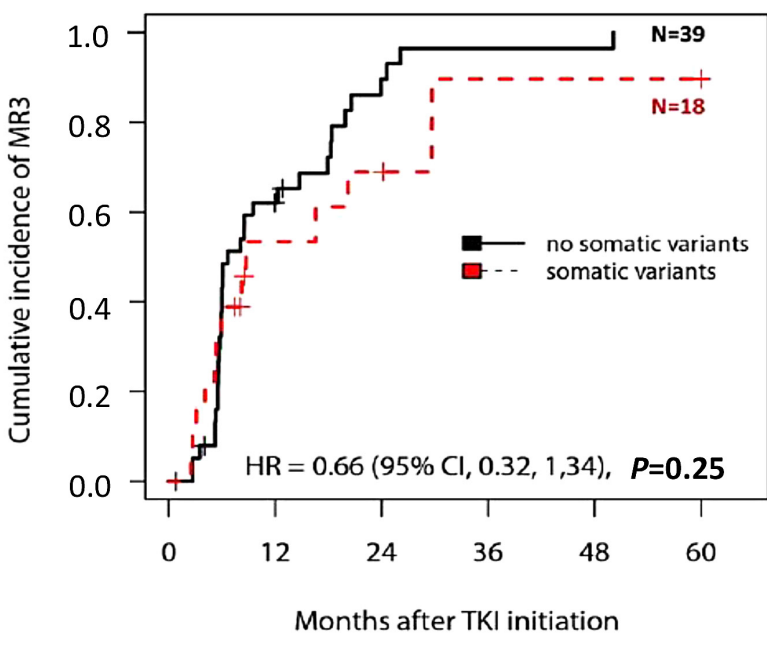

Figure 1. The use of $2^{\text {nd }}$-generation tyrosine kinase inhibitors (2G-TKI) can overcome the adverse effect of somatic mutation in epigenetic modifier genes in chronic myeloid leukemia (CML) patients. Incidence of achievement of major molecular response (MR3) following imatinib therapy (A) or 2G-TKI (B) according to the presence of somatic mutation in epigenetic modifier gene in newly diagnosed chronic phase CML patients. N: number; HR: hazard ratio; Cl: confidence interval.

neoplasms (MPN). ${ }^{6}$ For example, mutation profiles are used for the initial risk assessment of AML such as inclusion of several high-risk markers such as mutations in TP53, RUNX1, and ASXL1 and high allelic ratio of FLT3-ITD in the revised European LeukemiaNet risk stratification system. The decision for further consolidation therapy between allogeneic HCT versus conventional consolidation therapy can be made based on the ELN risk stratification system. ${ }^{7}$ In addition, there is growing evidence to suggest that NGS-based measurable residual disease status could predict long-term outcomes in AML patients after induction chemotherapy ${ }^{8}$ or after allogeneic HCT. ${ }^{9}$ Accordingly, a next-generation sequencing (NGS)-based genomic test is being incorporated into clinical practice in a diverse subtype of hematologic malignancies. So, what about in CML?

A series of previous studies have reported consistent findings on the genomics in CML; ${ }^{10-13}$ 1) somatic mutations, particularly those in epigenetic modification pathway, are recurrently identified in CML patients with a prevalence of approximately $30-40 \%$; 2) increasing frequency of the mutation was associated with TKI resistance and progression to advanced disease in comparison to optimal response to TKI therapy or chronic phase (CP) disease; 3) somatic mutation in epigenetic modification pathway has adverse prognostic implication. The ASXL1 mutation is most commonly detected mutation in CPCML patients with a prevalence of $9.7 \%$, while it was detected with a higher frequency of $15.1 \%$ in advanced phase CML patients. ${ }^{13}$ RUNX1 mutations and IKZF1 exon deletions were strongly associated with disease progression, given that it was more frequently detected in advanced phases. $^{13}$ With respect to adverse prognostic implications of mutation in epigenetic modification pathway, Kim et al. reported that patients carrying gene mutation in the epigenetic modification pathway showed inferior complete cytogenetic response at 12 months $(53 \%$ vs.
$79 \% ; P=0.02)$, major molecular response at two years (35\% vs. $62 \% ; P=0.04)$, and MR4.5 at three years $(26 \%$ vs. $47 \%$; $P=0.03) .{ }^{10}$ Although successful replication to confirm those findings is required with well-curated clinical outcome data, and inclusion of larger cohorts, the study of Nteliopoulos et al. ${ }^{14}$ presented in this issue of the Journal has validated the adverse prognostic impact of somatic mutation in the epigenetic modification pathway in the patients treated with imatinib. What is interesting in this study is that an adverse prognosis from a somatic mutation in the epigenetic modification pathway can be abrogated by the use of $2^{\text {nd }}$ generation TKI, which is very intriguing.

Nteliopoulos et al. ${ }^{14}$ have profiled genetic variants in epigenetic modifiers, including 71 candidate genes for predicting response to TKI therapy and progression to advanced disease. Out of 124 patients (including 62 patients treated with imatinib and 62 patients with $2^{\text {nd }}$ generation TKI), they reported that $30 \%$ of patients carry somatic variants in at least one of ASXL1, IKZF1, $D N M T 3 A, C R E B B P$, which is consistent with results from the previous studies. Non-responders have higher frequencies of somatic variants in those genes as compared to responders. When treatment outcomes were analyzed according to the TKI subtype and the presence of a mutation in epigenetic modifier gene, molecular response (MR3) in those with the mutation was significantly inferior to those without mutation when treated with imatinib $(P=0.048)$ (Figure 1$)$. On the other hand, the similar prognostic effect of a mutation in the epigenetic modifiers was not observed in patients treated with $2^{\text {nd }}$ generation TKI $(P=0.25)$ (Figure 1). Not only for MR3, but also analyses on other clinical end points showed that an adverse prognostic effect from mutations in epigenetic modifier genes are significantly reduced by the use of $2^{\text {nd }}$ generation TKI. The next step is validation and confirma- 


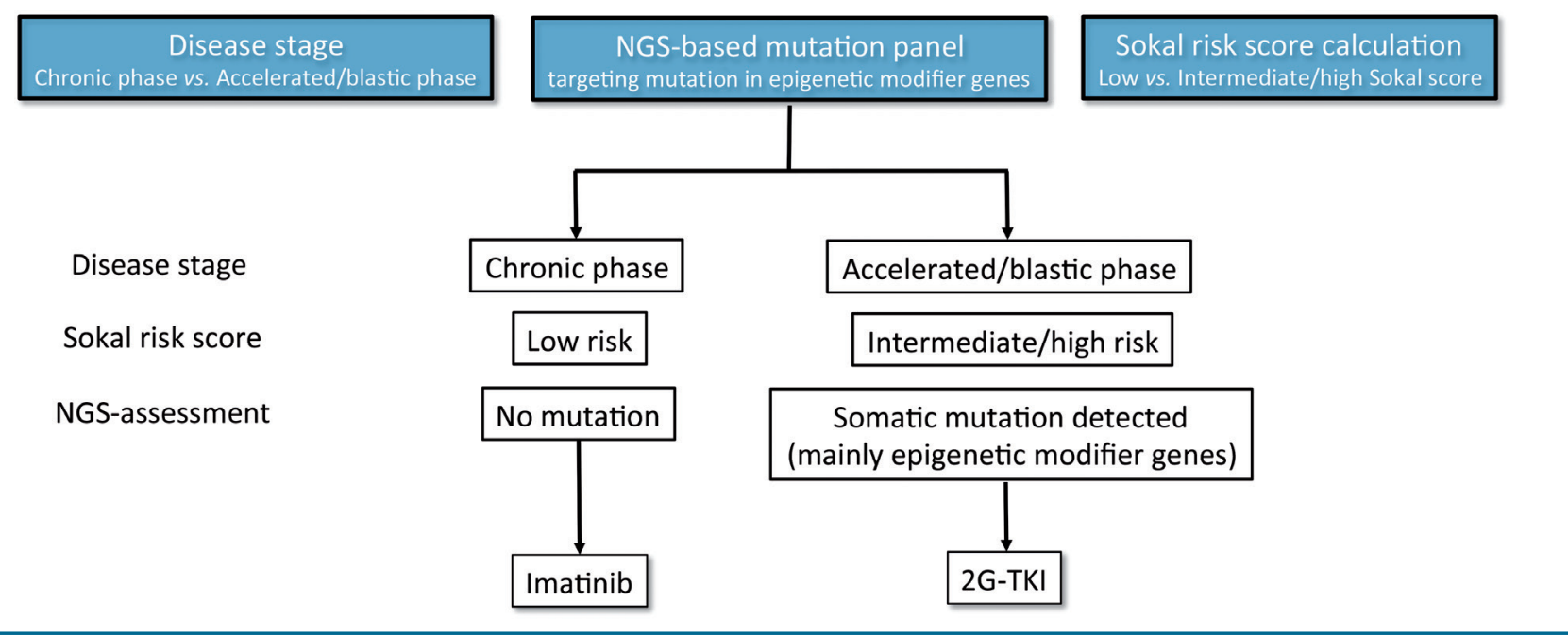

Figure 2. Treatment algorithm of chronic myeloid leukemia (CML) patients in future medicine incorporating next-generation sequencing (NGS)-based risk assessment and up-front tyrosine kinase inhibitor (TKI) drug selection.

tion of the finding before appropriate recommendations can be made, with the inclusion of NGS screening at initial diagnosis of CML. ${ }^{13}$ Moreover, clinical risk scores at diagnosis may inform the selection of patients for NGSbased screening. Figure 2 shows an example of future therapies incorporating NGS-based testing at diagnosis in CML management. Once the diagnosis of CML is made, the next step for risk assessment will include NGSbased risk assessment in addition to clinical disease staging (chronic phase vs. accelerated phase vs. blastic phase) or Sokal risk score calculation. In the case of advanced disease phase, intermediate to high Sokal risk score or those with a somatic mutation in epigenetic modifiers pathway such as ASXL1, DMNT3A, TET2 will be strong candidates for upfront therapy using the $2^{\text {nd }}$ generation TKI.

In the context of somatic mutation profile in CML, some questions remain: 1 ) what is the role of age-related clonal hematopoiesis in the development of cardiovascular toxicity following TKI therapy; 2) what is the role of somatic mutations in TKI switch for TKI resistant cases without carrying ABL1 kinase domain mutation; 3) what is the clinical relevance of somatic mutations with respect to treatment-free remission? Future studies are warranted to answer these questions so that somatic mutation profiles can be incorporated into future CML practice not only for upfront TKI drug selection but also during follow up with TKI therapy.

There is a limitation in the study by Nteliopoulos et al..$^{14}$ the study cohort did not consist of a consecutive set of patients. Thus, further study is strongly warranted to reach a clearer conclusion with a larger prospectively collected cohort. Upon successful validation of these data, this approach using NGS-based precision medicine will eventually be incorporated into a clinical algorithm of CML management such as future ELN recommendations. Precision medicine will soon be part of our practice even in CML.

\section{References}

1. Bower H, Björkholm M, Dickman PW, Höglund M, Lambert PC, Andersson TML. Life expectancy of patients with chronic myeloid leukemia approaches the life expectancy of the general population. J Clin Oncol. 2016;34(24):2851-2857

2. Hughes T, Deininger M, Hochhaus A, et al. Monitoring CML patients responding to treatment with tyrosine kinase inhibitors: Review and recommendations for harmonizing current methodology for detecting BCR-ABL transcripts and kinase domain mutations and for expressing results. Blood. 2006;108(1):28-37.

3. Baccarani M, Deininger MW, Rosti G, et al. European LeukemiaNet recommendations for the management of chronic myeloid leukemia: 2013. Blood. 2013;122(6):872-884

4. Pavlů J, Szydlo RM, Goldman JM, Apperley JF. Three decades of transplantation for chronic myeloid leukemia: What have we learned? Blood. 2011;117(3):755-763

5. Papaemmanuil E, Gerstung M, Bullinger L, et al. Genomic Classification and Prognosis in Acute Myeloid Leukemia. N Engl J Med. 2016;374(23):2209-2221.

6. Guglielmelli P, Lasho TL, Rotunno G, et al. MIPSS70: Mutationenhanced international prognostic score system for transplantation-age patients with primary myelofibrosis. J Clin Oncol. 2018;36(4):310-318.

7. Döhner H, Estey E, Grimwade D, et al. Diagnosis and management of AML in adults: 2017 ELN recommendations from an international expert panel. Blood. 2017;129(4):424-447.

8. Jongen-Lavrencic M, Grob T, Hanekamp D, et al. Molecular Minimal Residual Disease in Acute Myeloid Leukemia. N Engl J Med. 2018;378(13):1189-1199.

9. Kim T, Moon JH, Ahn J-S, et al. Next-generation sequencing based post-transplant monitoring of acute myeloid leukemia identifies patients at high risk of relapse. Blood. 2018;132(15):1604-1613.

10. Kim T, Tyndel MS, Kim HJ, et al. Spectrum of somatic mutation dynamics in chronic myeloid leukemia following tyrosine kinase inhibitor therapy. Blood. 2017;129(1):38-47

11. Schmidt M, Rinke J, Schäfer V, et al. Molecular-defined clonal evolution in patients with chronic myeloid leukemia independent of the BCRABL status. Leukemia. 2014;28(12):2292-2299.

12. Branford S, Wang P, Yeung DT, et al. Integrative genomic analysis reveals cancer-associated mutations at diagnosis of CML in patients with high risk disease. Blood. 2018;132(9):948-961.

13. Branford S, Kim DDH, Apperley JF, et al. Laying the foundation for genomically-based risk assessment in chronic myeloid leukemia. Leukemia. 2019;33(8):1835-1850.

14. Nteliopoulos G, Bazeos A, Claudiani S, et al. Somatic variants in epigenetic modifiers can predict failure of response to imatinib but not to second generation tyrosine kinase inhibitors. Haematologica. 2019;104(12):2400-2409. 